\title{
Development of Hybrid Avalanche Photo Detector and its Readout Electronics for the Belle II Aerogel RICH counter
}

\author{
S. Iwata ${ }^{*}, a$, I. Adachi ${ }^{b}$, K. Hara $^{b}$, T. Iijima $^{c}$, S. Iori $^{d}$, H. Kakuno ${ }^{a}$, R. Kataura ${ }^{e}$, H. \\ Kawai $^{f}$, T. Kawasaki ${ }^{e}$, T. Kobayashi ${ }^{e}$, S. Korpar ${ }^{g, h}$, P. Krizan $^{g, i}$, S. Nishida ${ }^{b}$, S. \\ Ogawa $^{d}$, R. Pestotnik ${ }^{g}$, L. Santelj ${ }^{b, g}$, M. Shoji $^{b}$, A. Seljak ${ }^{g, h}$, T. Sumiyoshi ${ }^{a}$, M. \\ Tabata $^{f, j}$, E. Tahirovic ${ }^{g}$, T. Uchida ${ }^{b}$, K. Yoshida ${ }^{a}$, and Y. Yusa ${ }^{e}$ \\ ${ }^{a}$ Tokyo Metropolitan University, Japan, \\ ${ }^{b}$ High Energy Accelerator Research Organization (KEK), Tsukuba, Japan, \\ ${ }^{c}$ Nagoya University, Japan, \\ ${ }^{d}$ Toho University, Japan, \\ ${ }^{e}$ Niigata University, Japan, \\ ${ }^{f}$ Chiba University, Japan, \\ g Jožef Stefan Institute, Ljubljana, Slovenia \\ ${ }^{h}$ University of Hawaii, Honolulu, Hawai \\ ${ }^{i}$ University of Maribor, Slovenia \\ ${ }^{j}$ University of Ljubljana, Slovenia \\ ${ }^{k}$ Japan Aerospace Exploration Agency (JAXA), Japan \\ E-mail: iwatadhepmail.phys.se.tmu.ac.jp
}

For the Belle II experiment at the superKEKB accelerator, we have been developing a proximity focusing ring imaging Cherenkov detector using a silica aerogel as a radiator (A-RICH). This counter is designed to be used at the forward end-cap region and to have pion/kaon separation with more than 4-sigma deviations at momenta up to $4 \mathrm{GeV} / c$. 144-channel Hybrid Avalanche Photo-Detector (HAPD) modules developed with Hamamatsu Photonics K.K. have been adopted as the photon detectors for the A-RICH. We started the mass production of the HAPD since 2013, and we are testing quality of the manufactured HAPDs. As for the readout of about 60,000 channels from the A-RICH, we developed an ASIC for the amplification and digitization of the signals from HAPDs. We had finished the mass production of the ASIC chips. Then we are testing all the ASIC chips before mounting them on the front-end boards attached to HAPDs. The data from several front-end boards will be merged into one board, and are sent to the Belle II central DAQ system. For this purpose, modules called "merger board" located inside the detector are under development. We are developing the firmware for the FPGA and testing it. In this report, current status of the development of HAPDs and readout electronics for the A-RICH are described.

Technology and Instrumentation in Particle Physics 2014,

2-6 June, 2014

Amsterdam, the Netherlands

\footnotetext{
* Speaker.
} 


\section{Introduction}

The Belle experiment [ $[\mathrm{d}]$ is one of the B-factory experiments with an $e^{+} e^{-}$collider for the $C P$ violation search in $B$ meson system. It finished in 2010, and the KEKB accelerator and the Belle detecter are being upgraded to the SuperKEKB and the Belle II, respectively [[]]. The Belle II experiment is planned to explore new physics beyond the standard model using high precision measurements of physics parameters. The charged particle identification (PID) in the large range of momenta plays an important role in high precision measurements. Especially, kaon and pion identification up to $4 \mathrm{GeV} / c$ at the forward end-cap region is needed to search for new physics in the flavor structure. For example, it is important to separate $B \rightarrow \rho \gamma$ and $B \rightarrow K^{*} \gamma$ decays because mesons produced in these modes are decayed into $\pi^{ \pm} \pi^{\mp}$ and $K^{ \pm} \pi^{\mp}$, respectively [四]. Therefore, identification of charged mesons is essential for precision measurements at the Belle II. In the endcap region of the Belle, a threshold-type Aerogel Cherenkov Counter (ACC) was used for PID. Its whose momentum range for $K / \pi$ separation was limited up to $2 \mathrm{GeV} / c$ and thus, it is not sufficient for requirement in the Belle II experiment. Then we have been developing the new PID device which can separate kaon and pion up to $4 \mathrm{GeV} / c$ for the Belle II forward end-cap region.

The place where the forward end-cap PID device will be set is under a magnetic field of $1.5 \mathrm{~T}$. In addition, the space is limited because depth of the room along the beam direction is $280 \mathrm{~mm}$. Therefore, a compact and high performance PID detector is needed. We are developing a proximity focusing ring imaging Cherenkov counter using a silica aerogel as a radiator, which is called Aerogel RICH (A-RICH). The aim of the counter is $K / \pi$ separation of more than $4 \sigma$ at $4 \mathrm{GeV} / c$ in the forward end-cap region. The principle of the A-RICH is shown in Fig. $\mathrm{W}$.

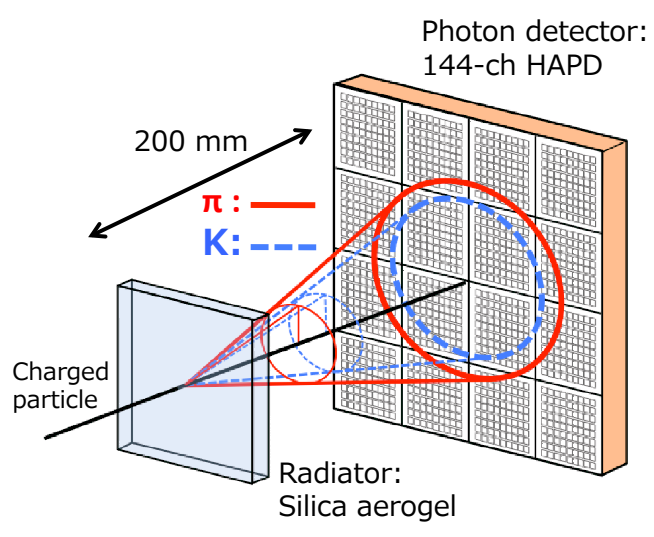

Figure 1: The principle of the $K / \pi$ identification of the A-RICH. The expansion length for Cherenkov photons from radiators to photon detector arrays is $200 \mathrm{~mm}$. Typical radius of Cherenkov ring is around $60 \mathrm{~mm}$ and the difference between radii of $K / \pi$ rings is around $5 \mathrm{~mm}$ at $4 \mathrm{GeV} / c$.

As the radiator, highly transparent silica aerogel, whose typical refractive index is about 1.05, is used. Cherenkov light is produced, while a charged particle is traveling through the silica aerogel radiator. The Cherenkov photons are detected as a ring image on a multi-pixel photon detector. From the radiation angle $\theta_{\mathrm{C}}$ calculated from the radius of the Cherenkov ring, particle identification 
is performed for the charged particle by the equation:

$$
m=\frac{p}{c} \sqrt{n^{2} \cos ^{2} \theta_{\mathrm{C}}-1}
$$

where $m$ is the particle mass, $p$ is the particle momentum obtained from other detectors, $c$ is the speed of light and $n$ is the refraction index of the aerogel. The difference of the Cherenkov angles for a pion track and a kaon track is expected to be $23 \mathrm{mrad}$ for $p=4 \mathrm{GeV} / c$ and $n=1.05$.

Position resolution of about $5 \times 5 \mathrm{~mm}^{2}$ is required for sufficient $K / \pi$ separation because the deference of radii of Cherenkov rings for kaon and pion is around $5 \mathrm{~mm}$ at the momentum of $4 \mathrm{GeV} / \mathrm{c}$ if the photon detector is located $200 \mathrm{~mm}$ downstream of the aerogel radiator. We have developed 144-ch multi-anode Hybrid Avalanche Photo Detectors (HAPDs) for the photon detectors. The HAPD has sufficient performance for the A-RICH, because (i) its pixel size is $4.9 \times 4.9 \mathrm{~mm}^{2}$, (ii) the detector has high sensitivity to single photon, (iii) it is tolerable to high magnetic field, and (iv) it has radiation hardness sufficient for the Belle II experiment.

Since we use 420 HAPDs for the Belle II detector, the readout electronics of HAPDs are necessary to read out over 60,000 channels at once. However the space available for the readout system is quite limited, about $5 \mathrm{~cm}$ in depth, due to the end-cap structure. Therefore, we have developed a readout system based on analog ASIC chips and the data merging board based on logic FPGA chips. The merging system, which is called "Merger", performs data merging, transmission to the central Belle II DAQ and controlling parameters in ASICs. I will report the status of development and mass production of the HAPD, readout ASICs and related electronics.

\section{The status of the HAPD}

We have been developing 144-ch HAPDs with Hamamatsu Photonics K.K. since 2002 for the A-RICH detector. An HAPD module is composed of a vacuum tube and four APD chips, where an APD is pixelated into $6 \times 6$ pads, resulting in 144 channels in total. Figure $\square$ shows a snapshot and structure of the HAPD. Principal specifications of the HAPD are summarized in Table $\square$.

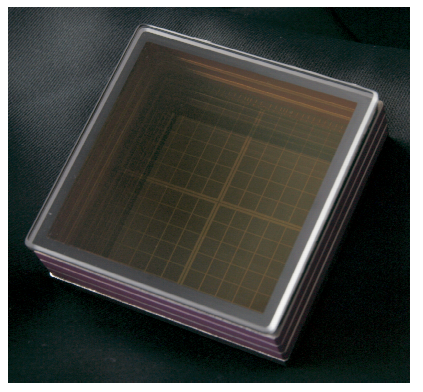

(a)

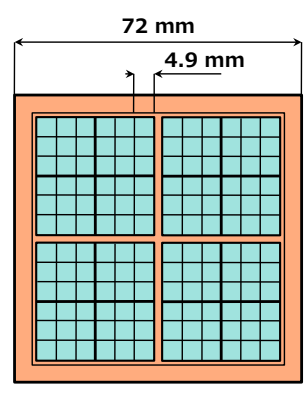

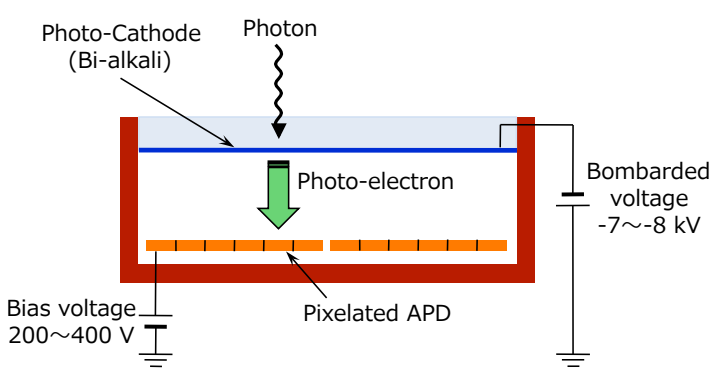

(b)

Figure 2: (a) The picture of outward and the design of the 144-ch HAPD. (b) A cross sectional view of the HAPD. Mechanism of electron amplification is also shown. There are two amplification mechanism of electron amplification. One is bombarded gain by electric field, and the other is avalanche gain in the APD.

In an HAPD module, photoelectrons are amplified inside the avalanche photo-diode (APD) in two steps (Fig. $\square($ b)). In the first step, each photoelectron accelerated with the high electric 
Table 1: HAPD specification

\begin{tabular}{cc}
\hline \# of pixels & $12 \times 12=144 \mathrm{ch}$ \\
Package size & $73 \times 73 \mathrm{~mm}^{2}$ \\
Pixel size & $4.9 \times 4.9 \mathrm{~mm}^{2}$ \\
Effective area & $65 \%$ \\
Capacitance & $80 \mathrm{pF}$ \\
Typical QE & $28 \%$ at $400 \mathrm{~nm}$ \\
Total gain & $\sim 7 \times 10^{4}$ \\
\hline
\end{tabular}

field produces about 1,700 electron-hole pairs. This process is called bombarded gain. Then the avalanche amplification occurs in the APD and this gain is around 40. As the result, total gain is around $7 \times 10^{4}$. We had confirmed the basic performance of the HAPD by bench test in our laboratory, and its results satisfy our requirements as shown in [3]].

The HAPD is also required to have the radiation hardness for 10-year Belle II operation. Especially, neutron flux of $10^{12}$ neutrons $/ \mathrm{cm}^{2}$ and $\gamma$-ray dose of $1 \mathrm{kGy}$ are expected in the end-cap region of Belle II.

In general, neutrons induce lattice defects in APD bulk region. They cause increasing leakage current through this region. Then, it is expected that noise from the HAPD increases due to leakage current and the single photon separation will be worse. In order to prevent the increasing noise, we changed thickness of the P-layer of the APD in the HAPD. Because leakage current from the $\mathrm{P}$-layer is more than it from the N-layer, increase (leakage) current is suppressed with thinner Player. In addition, the $\mathrm{P}^{+}$-layer which is the top part of the APD is better to be thinner to improve the bombardment gain. We had confirmed that thinner P-layer can suppress increase of leakage current by the neutron irradiation test in 2011 .

On the other hand, $\gamma$-ray irradiation causes charge-up around the structure on the APD surface. In particular, we found that the oxide layer on the APD was easily charged up by $\gamma$-rays. It causes breakdown at the place where withstand voltage is low. As the results, breakdown voltage is degraded than normal operational voltage, and avalanche gain will be lower. Therefore, we removed some oxide layer of an APD to prevent charge-up.

In 2012 and 2013, we performed irradiation tests to confirm that the manufactured HAPD with improved APD for hardness of neutrons and $\gamma$-rays have sufficient tolerance to neutrons and $\gamma$-rays expected at the Belle II [B]]. At first, we carried out the neutron irradiation test for them at the neutron beam line in J-PARC MLF (Ibaraki, Japan). Manufactured HAPDs were irradiated with $1.68 \times 10^{12}$ neutrons $/ \mathrm{cm}^{2}$ in maximum. After that (around three months later), we had the $\gamma$-ray radiation test with up to $1 \mathrm{kGy}$ at ${ }^{60} \mathrm{Co}$ facility in Nagoya university. As the result, we did not observe any breakdown in all samples. Therefore, we confirmed the HAPD has sufficient radiation hardness for neutrons and $\gamma$-rays expected in 10-year Belle II operation.

\section{Mass production}

The mass production of HAPD has already started from June 2013. We need 420 modules op- 
eration of the A-RICH and 30 modules for spares. The delivery of HAPDs started since September 2013, and scheduled to be ended in September 2014 as $30 \sim 40$ modules are delivered every month. However the schedule is delayed about a few months. Whole schedule of the HAPD production is modified and the completion of the delivery is extended in the end of 2014.

All HAPD modules, which are mass-produced and delivered from HPK, are objects of quality inspection. The inspection items are quantum efficiency $(\mathrm{QE})$ distribution and dead channel searching. In order to take QE distribution, we constructed the measurement system at KEK. The requirement for QE of HAPD modules is $28 \%$ in average and $24 \%$ at the minimum. The recent result from our QE measurement averages out $30.0 \%$ in 193 modules. To find dead channels, we performed following tests; measurement of leakage current, noise level in each channel, gain of output signal and 2-dimensional hit map. So far, we had checked 156 modules in total about these tests. Then, 134 modules are selected as the good sample, which has no dead channels or any other problems. It corresponds to $86 \%$ of a total. "Low quality" modules which have some dead channels are $3 \%$, modules under investigation are $10 \%$ and modules which were completely rejected by our requirement are $1 \%$, respectively.

In the quality inspection, we found some HAPD samples show noise in 2-dimensional hit map as in Fig. B (a). This hit map shows the hit rate of each APD pixel taken by scanning position of pulsed laser source uniformly. The expected hit map is as shown in Fig. B (b). However, huge noise hits appear on the left side in Fig. [3 (a). From the results of our investigation, this noise occurred after some light exposure. (e.g. when the door of a light tight box opens in order to place the HAPD.) Noise in most samples of this reason disappear in about 30 minutes after light exposure and applying operation voltages (Fig. B (b)). 10 of all noisy HAPDs, which we investigated, needed the standby time for about 30 minutes, and remained 7 HAPDs were needed for more than an hour.

Although the delivery schedule has some delay, the quality check of produced HAPDs goes well so far.

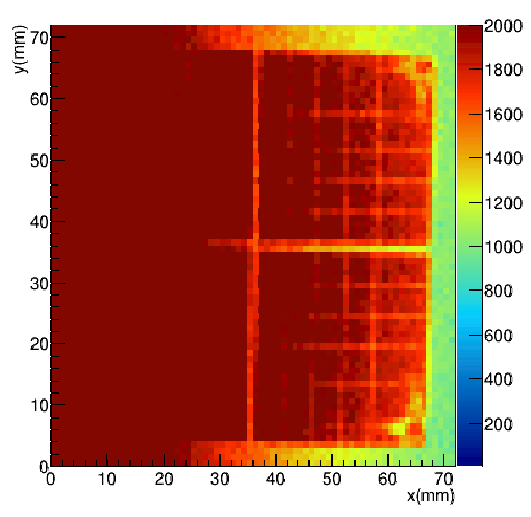

(a)

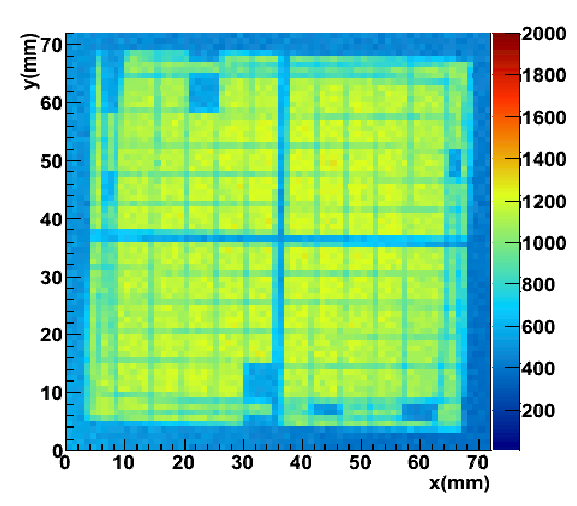

(b)

Figure 3: Results of the 2-dimensional laser injection test. Colors of each point mean the detection frequency of pulse laser light. (Original figures are colored.) (a) The sample which was affected by some noises. It has been taken just after bias voltages were applied. (b) The result of same sample, which had waiting for 30 minutes after applying bias voltages. A few channels look quiet because the readout electronics corresponding to that channel is dead. Noise in left side in (a) clearly disappeared. These results were obtained using our readout electronics. 


\section{The status of electronics}

\section{ASIC}

Requirement for our readout electronics are as bellows; (i) it has high-gain and low-noise amplifier, (ii) We need only the information on whether Cherenkov photons were detected at each channels or not and (iii) it (and also related circuit) has to be placed in the limited space within $5 \mathrm{~cm}$. In order to satisfy these condition, we developed original ASIC (Fig. 田 (a)). It has 36 channels, and main elements are charge-sensitive amplifier, shaper and discriminator. The shaper has variable shaping time. The power consumption per channel is about $3 \mathrm{~mW}$. Variable shaping time is important to reduce noise which is caused by neutron irradiation. Figure (b) and (c) show pulse height distribution of neutron irradiated HAPD with different shaping time. We confirmed that readout with shorter shaping time is able to reduce noise. Then, our ASIC is designed that shaping time can be set as short as $100 \mathrm{~ns}$.

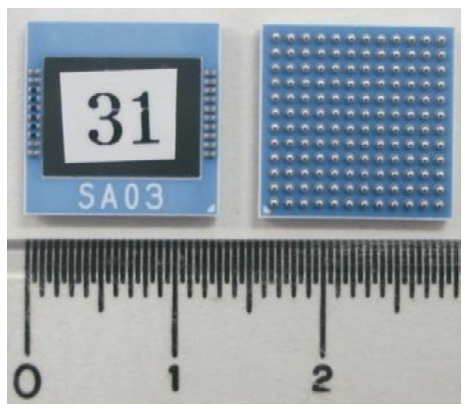

(a)

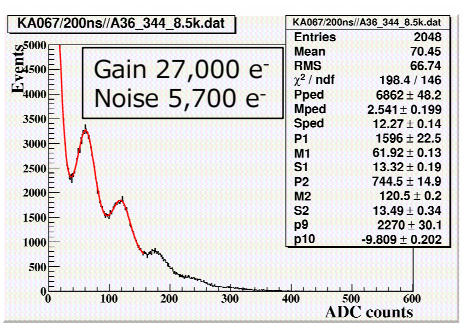

(b)

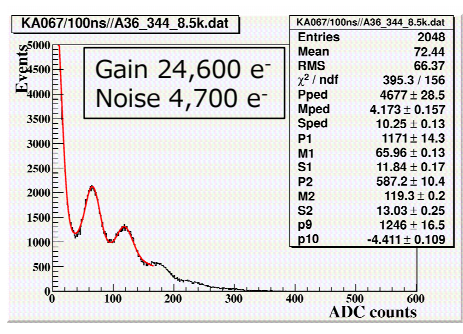

(c)

Figure 4: (a) Our readout ASIC (called "SA03"). (b) Pulse height distribution of a neutron irradiated HAPD. It was read out with $200 \mathrm{~ns}$ shaping time. (c) Pulse height distribution of the same HAPD with $100 \mathrm{~ns}$ shaping time. Value of gain and noise in these figures are calculated from distance between peaks and width of them.

ASIC production including packaging had already been finished. We need to select 1,680 good chips from the 2,500 mass-produced chips and thus, quality inspection is needed for this purpose. Quality check is mainly dead channel search and linearity of amplification gain in each channel. For this purpose, we developed the testing system. This system is able to measure 6 chips at once. We had measured 180 chips using this system. From this result, we found 47 dead channels in 30 chips, so far. The other 150 chips have no dead channel and any other problems. Test of remained chips and detailed test are on going.

\section{Front-end board}

Pictures of the front-end board are shown in Fig. 1. It has 4 ASIC chips, FPGA for treatment of digital signals, bias voltage connector for attached HAPD and temperature sensor chips [䧃][5]. The size of a front-end board is designed to fit an HAPD. We produced the first version of the hoard in 2011, and constructed the prototype A-RICH using them. Then, we succeeded in taking data and observing the ring image by test beam at CERN SPS [B]].

The final version of the front-end board is under designing. Mass production will start before the end of 2014. 


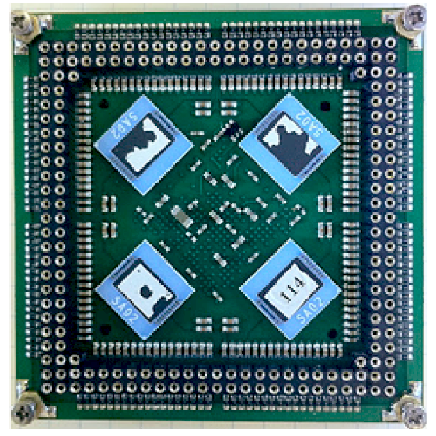

(a)

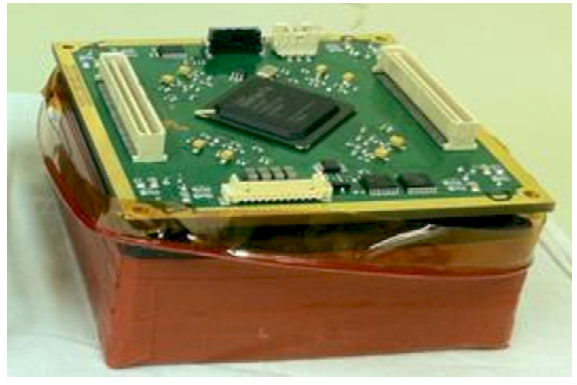

(b)

Figure 5: Pictures of the front-end board. (a) Connector side, (b) front-end board is attached on an HAPD.

\section{Merger system}

Total number of channels in the A-RICH is over 60,000 . Then, signal merging system is needed in order to suppress data size and the number of cables. We are developing the "merger board" for this purpose.

Figure 6 is shown the schematic of A-RICH DAQ flow using the merger board. A merger board merges hit-data from 5 or 6 front-end boards, and sends to the Belle II central DAQ through optical bus which is called Belle2Link. Data size suppression will be performed at FPGA on the merger board. In addition, the merger board will send parameters to FPGA chips on the front-end boards and distribute trigger signal and synchronized clock signal from the Belle II central system to the front-end board.

We produced a prototype of the merger board, and communication test is on going with it. The final version of the merger is under designing, mass production will start before the end of March in 2015 .

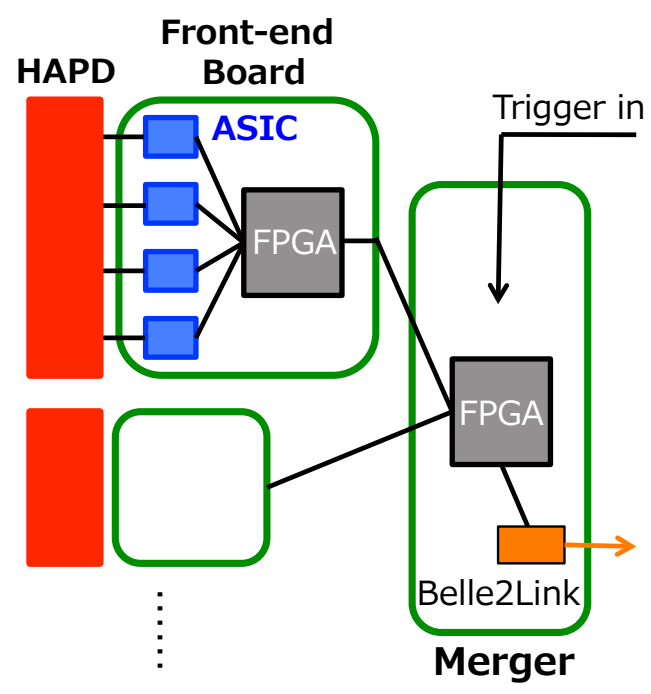

Figure 6: The schematic of A-RICH DAQ system. 


\section{Conclusion}

We are developing a proximity focusing aerogel ring imaging Cherenkov counter, which is called A-RICH, using 144-ch HAPDs for the Belle II experiment. We started to produce components of A-RICH since 2013. HAPDs as photon detector had been confirmed to satisfy with our requirements, therefore mass production of the HAPD is on going. And delivered modules are tested about quality of performance at KEK. So far, we had measured around 200 modules and selected around 130 modules as good sample. It corresponds to $86 \%$ out of total. We had finished the production of readout ASICs. These are under quality check. Designs of Front-end board and merger system on going for final version. Mass production of the front-end and the merger board will start before the end of 2014 and the end of March in 2015, respectively. All components will be ready in early 2015 .

We will start assembling the A-RICH counter in middle of 2015. After assembly of the counter, we are planning the cosmic-ray test for whole counter. Finally, installation of A-RICH in the Belle II is in 2015.

\section{References}

[1] A. Abashian et al. (Belle Collaboration), Nucl. Instr. and Meth. A479 (2002) 117.

[2] T. Abe et al. (Belle II Collaboration), arXiv:1011.0352v1[physics.ins-det], 2010.

[3] S. Iwata et al., 10.1109/NSSMIC.2012.6551358.

[4] A. Seljak et al., doi: 10.1088/1748-0221/6/01/C01083

[5] A. Seljak et al., doi: 10.1088/1748-0221/6/12/C12051 